\title{
Low-Dose Tramadol and Non-Steroidal Anti-Inflammatory Drug Combination Therapy Prevents the Transition to Chronic Low Back Pain
}

\author{
Kazuhide Inage ${ }^{1}$, Sumihisa Orita ${ }^{1}$, Kazuyo Yamauchi $^{1}$, Takane Suzuki ${ }^{2}$, Miyako Suzuki $^{2}$, Yoshihiro Sakuma ${ }^{3}$, \\ Go Kubota ${ }^{1}$, Yasuhiro Oikawa ${ }^{4}$, Takeshi Saino ${ }^{5}$, Jun Sato ${ }^{1}$, Kazuki Fujimoto ${ }^{1}$, Yasuhiro Shiga ${ }^{1}$, Koki Abe ${ }^{1}$, \\ Hirohito Kanamoto ${ }^{1}$, Masahiro Inoue ${ }^{1}$, Hideyuki Kinoshita ${ }^{1}$, Kazuhisa Takahashi ${ }^{1}$, Seiji Ohtori ${ }^{1}$ \\ ${ }^{1}$ Department of Orthopaedic Surgery, Graduate School of Medicine, Chiba University, Chiba, Japan \\ ${ }^{2}$ Department of Bioenvironmental Medicine, Graduate School of Medicine, Chiba University, Chiba, Japan \\ ${ }^{3}$ Department of Orthopaedic Surgery, National Hospital Organization Chiba Medical Center, Chiba, Japan \\ ${ }^{4}$ Department of Orthopaedic Surgery, Chiba Children's Hospital, Chiba, Japan \\ ${ }^{5}$ Department of Orthopaedic Surgery, Sainou Hospital, Toyama, Japan
}

\section{Study Design: Retrospective study.}

Purpose: To determine whether low-dose tramadol plus non-steroidal anti-inflammatory drug combination therapy could prevent the transition of acute low back pain to chronic low back pain.

Overview of Literature: Inadequately treated early low back pain transitions to chronic low back pain occur in approximately $30 \%$ of affected individuals. The administration of non-steroidal anti-inflammatory drugs is effective for treatment of low back pain in the early stages. However, the treatment of low back pain that is resistant to non-steroidal anti-inflammatory drugs is challenging.

Methods: Patients who presented with acute low back pain at our hospital were considered for inclusion in this study. After the diagnosis of acute low back pain, non-steroidal anti-inflammatory drug administration was started. Forty patients with a visual analog scale score of $>5$ for low back pain 1 month after treatment were finally enrolled. The first 20 patients were included in a non-steroidal anti-inflammatory drug group, and they continued non-steroidal anti-inflammatory drug therapy for 1 month. The next 20 patients were included in a combination group, and they received low-dose tramadol plus non-steroidal anti-inflammatory drug combination therapy for 1 month. The incidence of adverse events and the improvement in the visual analog scale score at 2 months after the start of treatment were analyzed.

Results: No adverse events were observed in the non-steroidal anti-inflammatory drug group. In the combination group, administration was discontinued in 2 patients (10\%) due to adverse events immediately following the start of tramadol administration. At 2 months, the improvement in the visual analog scale score was greater in the combination group than in the non-steroidal anti-inflammatory drug group $(p<0.001)$.

Conclusions: Low-dose tramadol plus non-steroidal anti-inflammatory drug combination therapy might decrease the incidence of adverse events and prevent the transition of acute low back pain to chronic low back pain.

Keywords: Spine; Low back pain; Tramadol; Anti-inflammatory agents, non-steroidal

Received Oct 13, 2015; Revised Dec 10, 2015; Accepted Dec 27, 2015

Corresponding author: Kazuhide Inage

Department of Orthopaedic Surgery, Graduate School of Medicine, Chiba University,

1-8-1 Inohana Chuo-ku, Chiba 260-8670, Japan

Tel: +81-43-226-2117, Fax: +81-43-226-2116, E-mail: kazuhideinage@yahoo.co.jp 


\section{Introduction}

The lifetime morbidity of low back pain has been reported to be at $80 \%$, and this malady has been shown to account for a large majority of the cases of locomotor diseases [1]. A diagnosis of low back pain can be established from imaging studies in approximately $20 \%$ of cases; however, non-organic cases outnumber organic cases, often leading to difficulty in mode of treatment [2]. A guideline for therapy in non-specific low back pain has not yet been established, and the socioeconomic loss from chronic low back pain has been shown to be significant [3]. Acute low back pain is curable in the early phase; however, chronic low back pain is resistant to treatment [4,5]. A recent analysis of low back pain showed that the transition of acute low back pain to chronic low back pain is particularly associated with elevated levels of certain cytokines.

Generally, inadequately treated early low back pain transitions to chronic low back pain in approximately $30 \%$ of affected individuals [6]. Therefore, early treatment is important in preventing the transition of acute low back pain to chronic low back pain. The administration of non-steroidal inflammatory drugs (NSAIDs) is effective for the treatment of low back pain at the early stage [2]. However, the treatment of low back pain that is resistant to NSAIDs is challenging. Tramadol, a drug indicated for non-cancerous pain, has recently been used for the treatment of low back pain. Tramadol is a non-narcotic central analgesic, and it acts as a $\mu$-opioid receptor agonist and activates the descending pain suppression pathways by inhibiting the reuptake of noradrenaline and serotonin. These mechanisms of action for tramadol differ from those of NSAIDs, and therefore, tramadol can effectively control chronic pain in patients who show a poor response to NSAIDs $[7,8]$. In addition, tramadol is expected to effectively control acute pain, as it is indicated for pain after tooth extraction [9].

Tramadol, however, is associated with a high incidence of adverse events, such as opioid-specific nausea and constipation, leading to drug withdrawal. A previous study indicated that dose titration from a low dose in the early phase can effectively prevent adverse events [10]. Based on this finding, we hypothesized that tramadol combination therapy with dose titration would reduce the incidence of adverse events and effectively control pain in patients who show a poor response to NSAIDs. Therefore, in the present study, we determined whether low-dose tramadol+NSAID combination therapy could prevent the transition of acute low back pain to chronic low back pain.

\section{Materials and Methods}

Patients who presented with the chief complaint of acute low back pain at our hospital were considered for inclusion in this study. Patients with a clear history of trauma and those who complained of leg pain, including radiating pain, were excluded. After the diagnosis of acute low back pain, NSAID administration was started.

Forty patients with a visual analog scale (VAS) score of $>5$ for low back pain 1 month after starting NSAID treatment were finally enrolled in the study. The first 20 patients were included in the NSAID group, and continued receiving NSAID therapy for 1 month. The next 20 patients were included in the combination group that received low-dose titrated tramadol+NSAID combination therapy (first dose: $25 \mathrm{mg}$ /day, up to $25 \mathrm{mg}$ every week) for 1 month. Additionally, laxatives and antiemetics were administered to all the patients to prevent major adverse events, such as constipation and nausea. This study was performed in accordance with the Declaration of Helsinki.

Chronic low back pain was defined as pain in the lower back lasting for more than 2 months. Thus, the incidence of adverse events and the improvement in the VAS score for low back pain at 2 months (chronic phase) after the diagnosis of acute low back pain were compared between the NSAID and combination groups. A $p$-value $<0.05$ was considered significant.

\section{Results}

\section{Group characteristics}

The characteristics of the patients in each group are presented in Table 1. No significant differences in sex, age, type of NSAID, history of diabetes, and VAS score at the initial visit were observed between the two treatment groups.

\section{Adverse events}

No adverse events were observed in the NSAID group. In the combination group, treatment was discontinued in 2 patients $(10 \%)$ because of adverse events ( 1 patient had nausea and 1 had constipation) immediately after starting 
Table 1. Characteristics of the patients in the non-steroidal inflammatory drug group and combination group

\begin{tabular}{lccc} 
Characteristic & NSAID group & Combination group & $p$-value \\
Sex (mal:femal) & $5: 15$ & $6: 14$ & 0.73 \\
Age (yr) & $69.9 \pm 13.1$ & $72.9 \pm 10.4$ & 0.43 \\
\hline Type of NSAIDS (celecoxib:loxoprofen) & $14: 6$ & $14: 6$ & 1.00 \\
Past history of diabetic & 0 & 0 & 1.00 \\
VAS at initial visit & $9.65 \pm 0.51$ & $9.85 \pm 0.49$ & 0.20 \\
\hline
\end{tabular}

NSAID, non-steroidal inflammatory drug; VAS, visual analog scale.

tramadol administration.

\section{VAS score}

At 1 month (with only the NSAID therapy), the mean VAS scores for low back pain were 6.7 in the NSAID group and 7.2 in the combination group $(p=0.07)$. However, at 2 months (after the NSAID group had received NSAID therapy and the combination group had received tramadol+NSAID therapy), the mean VAS score was significantly lower in the combination group than in the NSAID group (3.0 vs. 5.2, respectively; $p<0.001$ ) (Fig. 1).

\section{Changes in the VAS score}

At 1 month, the VAS score improved by $29.2 \%$ in the
NSAID group and $26.8 \%$ in the combination group $(p=0.10)$. However at 2 months, the improvement in the VAS score was significantly greater in the combination group than in the NSAID group ( $58.1 \%$ vs. $23.0 \%$, respectively; $p<0.001$ ) (Fig. 2).

\section{Discussion}

In the present study, adverse events were noted only in the combination group, with an incidence of 10\%. Previous studies have reported that the incidence of adverse events with tramadol administration was relatively high, occurring in $40 \%$ of cases [11]. Thus, the dose titration of tramadol in the present study, starting with a low dose (25 $\mathrm{mg}$ /day), obviated the incidence of adverse events, and this supports the findings of previous studies showing that

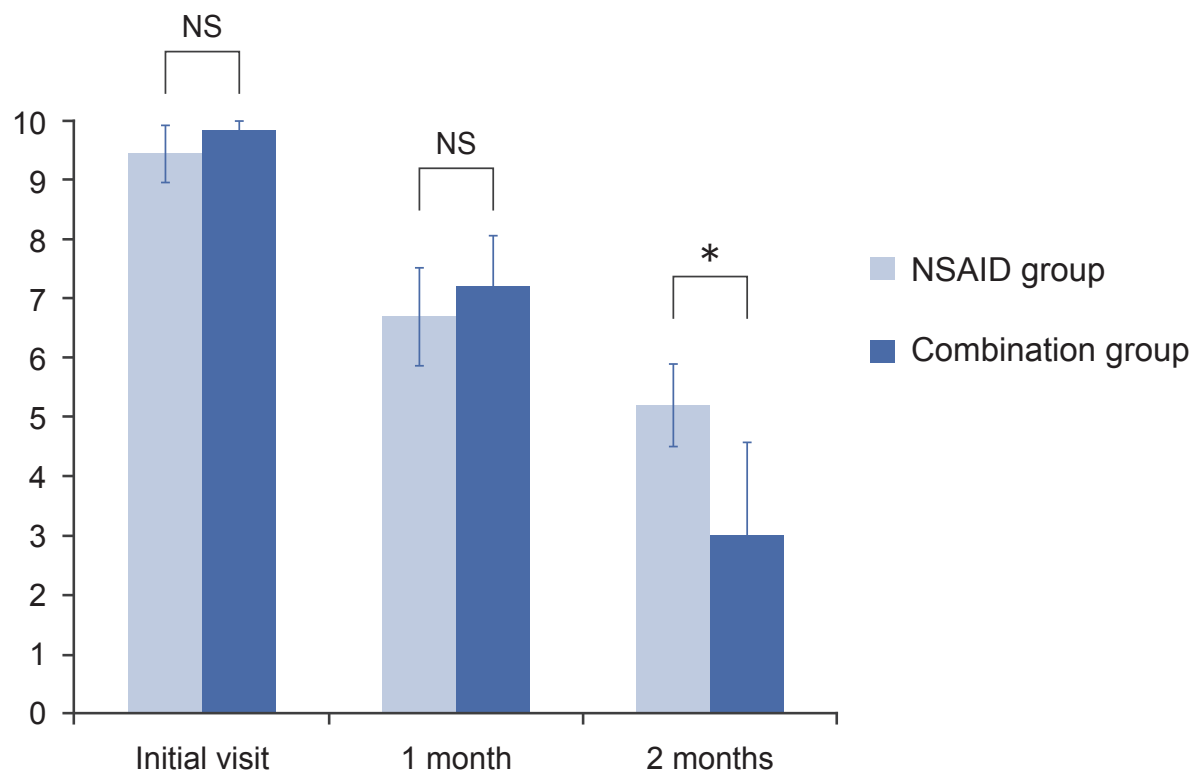

Fig. 1. Visual analog scale scores for low back pain. NSAID, non-steroidal inflammatory drug; NS, not significant. ${ }^{*} p<0.05$. 


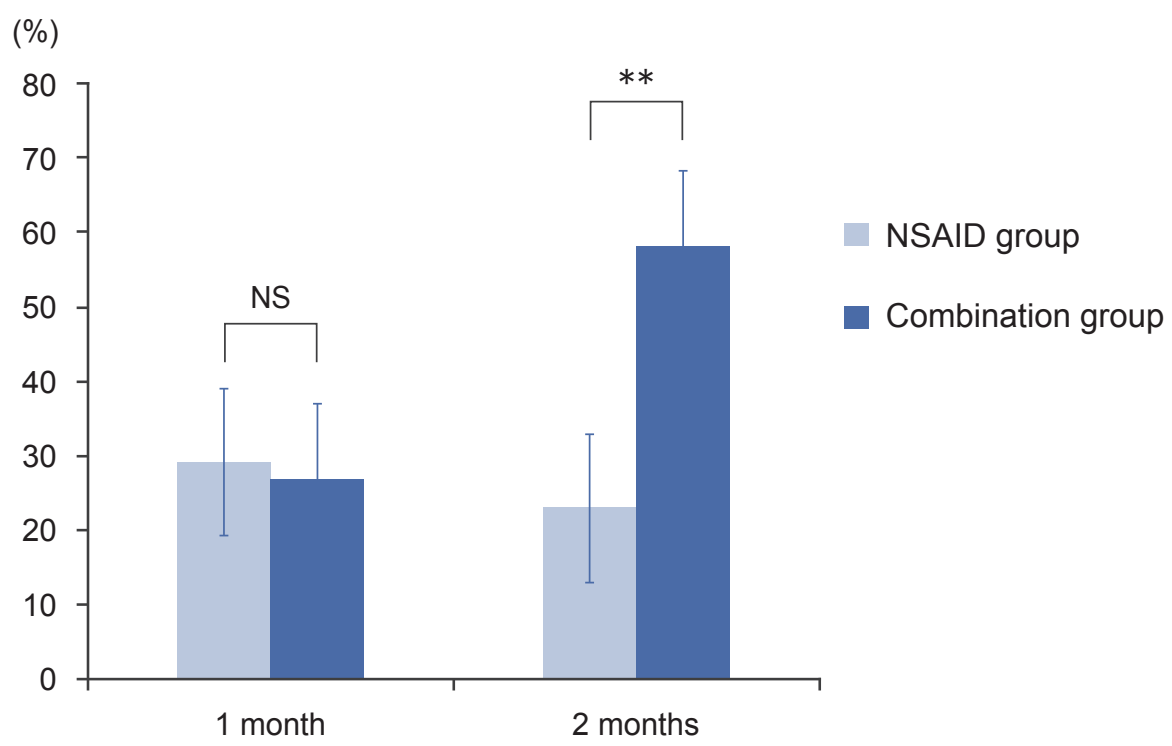

Fig. 2. Improvement in the visual analog scale scores for low back pain. NSAID, non-steroidal inflammatory drug; NS, not significant. ${ }^{* *} p<0.05$.

a dose titration regimen of tramadol significantly reduced the occurrence of adverse events. In addition, the prophylactic administration of laxatives and antiemetics might have also helped in reducing the occurrence of adverse events.

Studies have indicated that opioid-induced adverse events may have a genetic basis. In a recent gene analysis, adverse events developed in approximately $10 \%$ of patients [12]. The treatment approach used in the present study may help minimize adverse events in these individuals.

In the present study, the VAS score at 2 months after treatment was significantly lower and the improvement in the VAS score was significantly better in the combination group than in the NSAID group. These findings suggest that a transition to chronic low back pain may have been inhibited with tramadol combination therapy. Moreover, NSAIDs effectively treat acute low back pain [2].

Many factors other than inflammation are involved in chronic low back pain. Additionally, inflammatory pain may transition to neuropathic pain, and locomotor pain is mainly an inflammatory pain. When inflammation develops after tissue injury and degeneration, bradykinin, adenosine triphosphate, cytokine, and other factors may be released from lymphocytes and macrophages, leading to nociceptor activation and subsequent pain. Furthermore, a low pain threshold at the nerve endings and enhanced pain transmission at the posterior horn of the spinal cord bring about central sensitization, leading to neuropathic pain [13]. Pain associated with inflammation is limited at the very early part of the acute phase, whereas pain after this time involves multiple factors. Thus, the administration of only NSAIDs during the acute phase may have limitations. Tramadol exerts analgesic effects by activating descending pain suppression systems and influencing the opioid receptors through direct agonistic action. Therefore, the effectiveness of tramadol combination therapy may result from mechanisms that are not associated with NSAID therapy.

In the present study, tramadol combination therapy was started one month after the initial NSAID administration. Tramadol was not started initially with NSAIDs because insurance coverage of tramadol for non-cancerous pain is limited to cases that are difficult to treat with steroid analgesics. Tramadol has been indicated for pain after tooth extraction and has been shown to have an effect on acute pain [9]. Thus, we believe that combination therapy with tramadol and NSAIDs during the early phase allowed the achievement of good therapeutic effects against low back pain.

The present study had some limitations. Target illnesses, such as referred pain, were not assessed. Additionally, a tramadol only group was not included in the study. Moreover, pain was evaluated using only the VAS scoring, and the evaluation period was short ( 2 months). To overcome these limitations, we are planning to perform a additional 
study with a larger number of patients and a longer evaluation period.

\section{Conclusions}

Low-dose tramadol+NSAID combination therapy might decrease the incidence of adverse events and prevent the transition of acute low back pain to chronic low back pain.

\section{Conflict of Interest}

No potential conflict of interest relevant to this article was reported.

\section{References}

1. Andersson GB. Epidemiology of low back pain. Acta Orthop Scand Suppl 1998;281:28-31.

2. Deyo RA, Rainville J, Kent DL. What can the history and physical examination tell us about low back pain? JAMA 1992;268:760-5.

3. Suka M, Yoshida K. The national burden of musculoskeletal pain in Japan: projections to the year 2055 . Clin J Pain 2009;25:313-9.

4. van Tulder M, Becker A, Bekkering T, et al. Chapter 3. European guidelines for the management of acute nonspecific low back pain in primary care. Eur Spine J 2006;15 Suppl 2:S169-91.

5. Gurcay E, Bal A, Eksioglu E, Hasturk AE, Gurcay AG, Cakci A. Acute low back pain: clinical course and prognostic factors. Disabil Rehabil 2009;31:840-5.

6. Buchheit T, Van de Ven T, Shaw A. Epigenetics and the transition from acute to chronic pain. Pain Med 2012;13:1474-90.

7. Mullican WS, Lacy JR; TRAMAP-ANAG-006 Study Group. Tramadol/acetaminophen combination tablets and codeine/acetaminophen combination capsules for the management of chronic pain: a comparative trial. Clin Ther 2001;23:1429-45.

8. Zhang W, Moskowitz RW, Nuki G, et al. OARSI recommendations for the management of hip and knee osteoarthritis, Part II: OARSI evidence-based, expert consensus guidelines. Osteoarthritis Cartilage 2008; 16:137-62.

9. Kanto D, Salo M, Happonen RP, Vahlberg T, Kanto J. Tramadol premedication in operative extraction of the mandibular third molar: a placebo-controlled crossover study. Acta Odontol Scand 2005;63:43-9.

10. Choi CB, Song JS, Kang YM, et al. A 2-week, multicenter, randomized, double-blind, double-dummy, add-on study of the effects of titration on tolerability of tramadol/acetaminophen combination tablet in Korean adults with knee osteoarthritis pain. Clin Ther 2007;29:1381-9.

11. Pascual ML, Fleming RR, Gana TJ, Vorsanger GJ. Open-label study of the safety and effectiveness of long-term therapy with extended-release tramadol in the management of chronic nonmalignant pain. Curr Med Res Opin 2007;23:2531-42.

12. Angst MS, Phillips NG, Drover DR, et al. Pain sensitivity and opioid analgesia: a pharmacogenomic twin study. Pain 2012;153:1397-409.

13. Im HJ, Kim JS, Li X, et al. Alteration of sensory neurons and spinal response to an experimental osteoarthritis pain model. Arthritis Rheum 2010;62:29953005. 